\title{
Recursos informacionales, desarrollo endógeno y participación ciudadana en la Gestión Pública Local*
}

\author{
Fernández, Norcka** \\ Belloso, Nora*** \\ Delgado, Francys ${ }^{\star \star \star \star}$
}

\section{Resumen}

Partiendo de la premisa que el gobierno municipal debe propiciar la cooperación comunitaria con información veraz, clara y oportuna para incentivar el desarrollo local, se realizó esta investigación de tipo documental cuyo propósito fue explorar los recursos informacionales, el desarrollo endógeno y la participación ciudadana, elementos fundamentales en la gestión pública local. Los resultados evidenciaron a) Los recursos informacionales ayudan al cumplimiento de los objetivos y tareas del municipio b) la participación ciudadana se hace presente en las gestiones asociadas a la implementación de acciones públicas y c) la gestión pública local actúa como gestor democrático de la inclusión de los individuos a fin de favorecery promover el desarrollo de sus comunidades. Se concluye que estos elementos sirven de apoyo al proceso de la gestión pública local, permitiendo así el cumplimiento de su misión a través de la generación y administración de recursos informacionales que impulsen un desarrollo sustentable a través de los aportes de la ciudadanía.

Palabras clave: Desarrollo endógeno, participación ciudadana, recursos informacionales, gestión pública local.

Recibido: 13-06-07. Aceptado: 05-05-08

* Esta investigación representa un anticipo del proyecto "Recursos Informacionales, participación Ciudadana en la Gestión Pública Municipal” financiado por el CONDES.

** Dra. en Ciencias Gerenciales PPI adscrita al Centro de Estudios de la Empresa, profesora Titular de la Universidad del Zulia. E-mail: norkalibertad@gmail.com. Telf: 0416-6606770.

*** Mg. en Gerencia de Empresas PPI adscrita al Centro de Estudios de la Empresa. Profesor Asociado de La Universidad del Zulia. E-mail: nbellosor@yahoo.es. Telf: 0416-8615832.

**** Dra. en Ciencias Mención Investigación, Investigadora PPI adscrita al Centro de Estudios de la Empresa. Prof. Titular de la Universidad del Zulia.

E-mail: fradel16@yahoo.com. Telf: 0414-3628975. 
Recursos informacionales, desarrollo endógeno y participación ciudadana...

Fernández, Norcka; Belloso, Nora y Delgado, Francys

\title{
Information Resources, Endogenous Development and Citizen Participation in Local Public Management
}

\begin{abstract}
Starting from the premise that municipal government should favor community cooperation using true, clear and opportune information in order to motivate local development, a documentary-type study was carried out whose purpose was to explore informational resources, endogenous development and citizen participation, fundamental elements in local public management. Results indicated: a) informational resources help to fulfill the objectives and tasks of the municipality; b) citizen participation is present in management associated with implementing public actions; and c) local public management acts as a democratic agent for including individuals in order to favor and promote development of their communities. Conclusions are that these elements serve as support for the local public management process, allowing its mission to be fulfilled through the generation and administration of informational resources that drive sustainable development through citizen contributions.
\end{abstract}

Key words: Endogenous development, citizen participation, informational resources, local public management.

\section{Introducción}

La gestión municipal exige la mejor utilización de los recursos financieros, humanos e informacionales en pro de brindar bienestar a los ciudadanos; y es a través de esta gestión que la participación ciudadana se hace sentir, cuando el hombre tiene la oportunidad de contribuir, aportar e intervenir con su entorno municipal hacia el desarrollo de su comunidad.

El modelo de desarrollo endógeno representa un proceso de cambio, a través del cual se logra progreso, bienestar común, donde el hombre es el centro. Éste con sus necesidades, problemas, saberes, deseos de mejorar su nivel de vida y el poder municipal, representan agentes motivadores y estimulantes de estos cambios.

En este sentido, la información transmitida por los entes locales representa el elemento vinculante que hace posible la relación tridimensional entre el municipio, la participación ciudadana y el desarrollo endógeno. Asimismo los recursos informacionales pueden convertirse en productos electrónicos, digitalizables (texto, imagen, voz) e igualmente utilizar diversas vías, tecnológicas o físicas, que prestarían un apoyo importante a la comunidad.

Con la utilización eficiente de los recursos informacionales por parte del poder municipal, se les brindaría a los ciudadanos la información necesaria para facilitar su participación efectiva a través de la consolidación de una sociedad democrática, libre, solidaria y protagónica, así como fomentar diversas formas de organización social. Para lograr que la información se convierta en un recurso que permita la participación ciudadana, se debe llevar a cabo un proceso que implica el desarrollo de sistemas para el manejo y tratamiento de los recursos de informa- 
ción que, una vez soportados sobre una infraestructura tecnológica, permitan la agilidad y facilidad de acceso necesaria para la toma de decisiones.

Alcanzar el desarrollo de una cultura informacional dentro de la gestión pública, que permita el conocimiento de los conceptos, datos e información como recursos fundamentales en el desarrollo local, implicaría la utilización de las tecnologías de la comunicación e información (TIC), la administración de los recursos humanos y el desarrollo de los sistemas de información.

Sánchez y Graña (2000) y Primera, López y Montes de Oca (1999), indican que la conducción de los recursos informacionales en la generación de cambios significativos del comportamiento social, económico y cultural en contextos locales satisfacen los requerimientos específicos de desarrollo del municipio o nación.

Por lo tanto, los recursos informacionales en la gestión pública representan una fuente fundamental de insumos en materia administrativa, técnica, jurídica, política y económica, entre otras, que sirve para la generación de nuevos servicios y productos con valor agregado para la comunidad, estos recursos pueden ser aprovechados por su potencial informativo en beneficio de las comunidades locales y de la gestión pública en si misma.

El objetivo del presente trabajo fue explorar la vinculación entre el desarrollo endógeno y la participación ciudadana con los recursos informacionales y como estos elementos afectan significativamente en la gestión pública local. Para los fines de este estudio se realizó una investigación de tipo documental, basada en la revisión de fuentes de información elec- trónicas, textos y publicaciones periódicas, así como el análisis de enfoques conceptuales sobre las tres variables mencionadas anteriormente, a fin de realizar un estudio teórico sobre las vinculaciones existentes entre ellas.

\section{Recursos Informacionales. Reflexiones teóricas.}

Para definir lo que significa recursos informacionales, se partirá de las siguientes conceptualizaciones: recursose información.

Los recursos han sido definidos por algunos autores (Ponjuán, 1998; Cornella, 2003) como los elementos necesarios, tanto tangibles como intangibles, para que una organización cumpla con sus objetivos. Un recurso es, entonces, un elemento necesario para la actividad desarrollada en una organización.

Currás (2001) ha determinado que la información es un recurso elaborado a partir de los datos contenidos en los documentos para su subsiguiente utilización. Vale decir que la información es un elemento necesario para el desarrollo social, económico, político, cultural, entre otros. La información como recurso es el elemento imprescindible para el funcionamiento de las organizaciones.

En este mismo orden de ideas, Ranguelov (2004), indica que toda información, en el ámbito organizacional, persigue el objetivo principal de ayudar a la toma de decisiones, que se sistematiza para dar soporte adecuado a las estrategias de la empresa, proporcionando a todos los niveles de la organización la información necesaria para controlar las actividades de la misma. Asimismo, la infor- 
Recursos informacionales, desarrollo endógeno y participación ciudadana... Fernández, Norcka; Belloso, Nora y Delgado, Francys

mación se utiliza como un recurso corporativo que se planifica, gestiona y controla para que pueda ser más efectivo para la organización.

Por otra parte y desde un punto de vista genérico, la información ha sido enfocada por algunos autores (Coutin, 2005; Ponjuán, 1998 y Esteban, 2005) como un conjunto de datos ordenados de manera significativa, que relacionan la manera como suceden las cosas, hechos, fenómenos y cómo se vinculan a su vez con otros; permitiendo conocer situaciones, resolver problemas y satisfacer intereses.

Asimismo, Martínez (2000) define información como el proceso previo a la comunicación que convierte algo real (un hecho, una idea o una opinión) en algo comunicable.

Igualmente, Goñi (2000: 3) concibe el término información bajo el concepto de "significado que otorgan las personas a las cosas. Los datos se perciben a través de los sentidos, estos los integran y generan la información necesaria para el conocimiento que permite tomar decisiones para realizar las acciones cotidianas que aseguran la existencia social". Estos datos, a su vez son transmitidos a otras personas para su uso y asimilación.

De modo que, la información es el proceso que permite conocer lo que otros piensan a través de la comunicación bien sea oral, escrita, gesticular o mediante símbolos o signos. Esta información se puede utilizar como un recurso para llevar a cabo actividades encaminadas, no sólo al desarrollo de las organizaciones, sino de las sociedades.

Considerando el aspecto anterior, Cornella (2003) señala que la información es un recurso intangible necesario para el perfeccionamiento de una organización, puesto que tanto la información formal (en soporte físico o electrónico) como la informal (la experiencia y las habilidades de las personas) permiten desarrollar la capacidad de aprender a manipular otros recursos, de comunicarse entre sí las personas que forman parte de la organización y de tener métodos adecuados para la toma de decisiones.

La información no equivale a conocimiento (Coutin, 2005; Esteban, 2005), pero éste se hace posible a partir de aquélla y por lo mismo se convierte en un recurso vital para el desarrollo científico, tecnológico, artístico y cultural de la humanidad, donde, a pesar de la heterogeneidad que la caracteriza, se enfrentan problemas comunes para cuya solución la información es esencial e imprescindible.

Cuando se plantea que la información es un recurso, se le compara y se le ubica a un mismo nivel con otros recursos ya conocidos (humanos, financieros, tecnológicos, etc.). Por lo tanto, la información no debería ser considerada sólo como un apoyo o soporte, sino como uno de los principales recursos o activos de la organización. Es decir que un recurso informacional juega un papel decisivo en las organizaciones. Este aspecto es apoyado por Ponjuán (1998) cuando menciona que los recursos informacionales, permiten aprovechar, utilizar y trabajar los demás recursos de una organización permitiendo su desarrollo adecuado.

De igual manera, Sánchez y Rodríguez (2000), definen a un recurso informacional como aquel que una organización recibe, descubre y registra en cualquier forma para su posterior vínculo con otros hechos o fenómenos. 
Tomando en cuenta lo mencionado por los autores, podemos decir que los recursos informacionales tienen las siguientes características esenciales:

- Se necesitan para la toma de decisiones.

- Son imprescindibles para el funcionamiento de las organizaciones.

- Llevan un proceso sistemático para su aprovechamiento.

Por lo tanto, son considerados como un elemento importante para el desarrollo de cualquier organización, pues incrementa su desarrollo. Sin embargo, es importante destacar que, para el aprovechamiento de estos recursos, es imprescindible que hayan pasado por un proceso que facilite la utilización de la información que ellos ofrecen.

Asumimos que los recursos informacionales son los elementos necesarios que una organización utiliza para la toma de decisiones a través de los datos ordenados significativamente que ellos ofrecen, permitiendo conocer situaciones, resolver problemas y satisfacer intereses organizacionales. Dichos datos tienen que ser procesados de tal forma que puedan recuperarse eficientemente. Estos recursos pueden ser aquellos documentos producto de una actividad administrativa o aquellos que puedan ser generados con el fin de difundir una información. Pero lo importante es organizarlos como un todo que permita localizar la información requerida en el momento preciso.

Desde el punto de vista de Peña (2004), los recursos informacionales que facilitan la gestión en una organización serían: leyes, reglamentos, planes, programas, proyectos, ordenanzas y decre- tos. Sin dejar de mencionar aquellas instrucciones en comunicaciones escritas $u$ órdenes verbales, las que son consideradas como recursos informacionales generados en la gestión administrativa para expresar directrices y lineamientos sin seguir una política de información establecida.

Frecuentemente, las concepciones tradicionales de recursos de información se han limitado a documentos en papel o a las fichas; pero también, con el uso de las nuevas tecnologías, se han usado como recursos los respaldos en cintas o medios ópticos, así como los sitios y portales Web, que se han convertido en una fuente fundamental de información para empleados de empresas, clientes o usuarios potenciales y reales, asociados de una organización y la comunidad en general.

Dentro de los distintos tipos de recursos informacionales que suelen existir en las organizaciones, Martínez (2001) y Martín (2001), plantean los siguientes: de gestión, factuales o estadísticos y documentales.

En consonancia con esta tipología de recursos informacionales, Valdés (2000) menciona que, aunque estos recursos son la base de muchos procesos en toda organización, generalmente más del 80 por ciento de la información de la misma se encuentra en la forma de documentos impresos y, en la actualidad, los recursos informacionales son los de más rápido crecimiento, con un aumento de hasta un 200 por ciento al año en algunos sectores sociales. Lo cual implica que deberían estar procesados para poder recuperarse oportunamente.

Asimismo, un recurso informacional, no sólo incluye a la información en sí 
Recursos informacionales, desarrollo endógeno y participación ciudadana... Fernández, Norcka; Belloso, Nora y Delgado, Francys

misma, sino a las unidades de información, definidas por Guerrero (2004) como aquellas entidades con la capacidad de crear, adquirir, suministrar, procesar, almacenar o diseminar información, las cuales contienen todas las fuentes, servicios, sistemas y tecnologías asociadas a la satisfacción de las necesidades de información.

A las entidades que hace referencia el autor son los servicios de información, como las bibliotecas, los centros de documentación e información y los archivos. Estas instituciones tienen mucho en común, puesto que todas tienen como actividad primordial servir de intermediarias entre quienes generan la información y quienes la utilizan.

De la misma manera, en el artículo 110 de la Constitución de la República Bolivariana de Venezuela (1999), se reconocen los servicios de información como necesarios por ser parte de los "instrumentos fundamentales para el desarrollo económico, social y político del país, así como para la seguridad y soberanía nacional" (Asamblea Nacional, 1999).

De allí que se considere que, los recursos informacionales son un elemento importante para cualquier sociedad, forman parte de aquellos recursos fundamentales para el desarrollo integral de un país, por lo que se ha hecho siempre empeño en el fortalecimiento de los sistemas nacionales de información, a fin de que los ciudadanos puedan disponer tanto de fuentes nacionales como extranjeras, que permitan la participación de los mismos en el desarrollo endógeno local.

A nuestro juicio, los recursos informacionales, producen cambios, no sólo económicos, sino organizativos, cultura- les, educativos, y de personalidad, al impactar en la forma de pensar, percibir y entender la realidad y generar, de esta forma conocimientos. Por lo cual, es importante intercambiar estos recursos e integrarlos con los esfuerzos que actualmente se adelantan por parte de la gestión pública a través de la participación ciudadana.

Cabe destacar que, en la era de la información, ha crecido el reconocimiento de los distintos valores significativos de los recursos informacionales. Para Cornella (2003), ha aumentado el consumo de información, sea comprando publicaciones periódicas, a través de la televisión o con la utilización de Internet, y cada vez hay más actividades de intercambio de información en nuestro entorno. El manejo de recursos informacionales constituye una parte importante de las actividades personales y de la mayoría de las sociedades, organizaciones, empresas o instituciones.

La información se ha transformado así en un recurso cada vez más indispensable para el éxito de cualquier organización y es un recurso necesario para el desarrollo de las naciones y de su integración regional y mundial. Este éxito se alcanzaría a través de la aplicación de políticas de información dentro de la gestión pública que permitirían el aprovechamiento de los recursos informacionales con un fin productivo para la sociedad. Aseveración ésta refrendada por Primera, Montes de Oca y Fernández (2002:10), cuando señalan que "la información es un elemento que favorece la integración del ciudadano a la dinámica social y contribuye a su calidad de vida". 
Partiendo de esta premisa, se infiere que los recursos informacionales en la gestión pública se han convertido en un medio clave para el desarrollo de la participación ciudadana, en la búsqueda del cambio social que incluye la transformación de la infraestructura técnica y organizativa de su localidad.

\section{Desarrollo endógeno: un modelo integrador entre el Estado y la sociedad.}

Actualmente el desarrollo endógeno se ha convertido en un tema de mucho interés nacional .Por consiguiente, es necesario su conceptualización a objeto de tener varias definiciones que soporten el discernimiento e interpretación como una propuesta de transformación social que va a permitir la integración de entes importantes tales como el estado y la participación ciudadana vinculados con los recursos que genere el estado y fundamentalmente los informacionales como producto de índole cultural, que van a permitir la generación de conocimiento y transformación en pro del desarrollo endógeno.

Considerando lo expuesto, se han tomado en cuenta lo señalado por los autores como Vásquez (2006); Sunkel (1991), Boisier (citado por Martínez, 2006) y el Instituto Latinoamericano y del Caribe de Planificación Económica y Social (ILPES 2006).

Para Vásquez (2006), el desarrollo endógeno puede entenderse como un proceso de crecimiento económico y cambio estructural por la comunidad local, utilizando el potencial de desarrollo que conduce a la mejora del nivel de vida de la población.
En este sentido, representa la capacidad de una determinada región para generar bienestar colectivo, partiendo de sus recursos naturales disponibles, e incorporando los diferentes cambios que le permitan crecer con la participación de los diferentes actores locales y sus correspondientes conocimientos tecnológicos y culturales.

Paralelamente, el Instituto Latinoamericano y del Caribe de Planificación Económica y Social, ILPES (2006), lo definen como un proceso reactivadory dinamizador de la economía local, que mediante el aprovechamiento eficiente de los recursos endógenos, existentes en una determinada zona, es capaz de estimular el crecimiento económico, crear empleo y mejorar la calidad de vida.

Mientras que en el planteamiento de Sunkel (1991), se dejan ver opciones para orientar la industrialización "desde adentro" hacia determinados mercados internos y externos, prioritarios en la estrategia de desarrollo de largo plazo, en los cuales nuestros países posean o puedan adquirir niveles de excelencia relativa que les garanticen una sólida inserción en la economía mundial.

Para Boisier (citado por Martínez, 2006), este desarrollo constituye un enfoque humano, que le permite al hombre su transformación, potenciarse a si mismo para llegar a ser una verdadera persona humana. El desarrollo no lo hace nadie sino el hombre, su individualidad y en su sociabilidad. Este autor está ganando fuertemente en la idea de que el desarrollo endógeno es una fuerza espiritual, es la voluntad de superar las necesidades en un ámbito de encuentros de práctica de valores, cuyo principal requerimiento 
Recursos informacionales, desarrollo endógeno y participación ciudadana... Fernández, Norcka; Belloso, Nora y Delgado, Francys

es lo cognitivo, un individuo abierto al cambio permanente. En eso está basado su enfoque en el conocimiento para la acción territorial.

A nuestro juicio, el desarrollo endógeno implica una transformación social, cultural y económica de la región, que se relaciona directamente con la utilización eficiente de los recursos naturales en productos que se pueden consumir, distribuir e incluso exportar, basadas en relaciones equitativas de producción, respeto al medio ambiente, donde los actores económicos y sociales se integran con las instituciones locales formando un sistema denso de relaciones que incorporan los valores de la sociedad en el proceso de desarrollo.

Al observar las definiciones expuestas, en cada una de ellas se destacan elementos que describen el desarrollo endógeno como un proceso naciente de adentro hacia fuera, y donde el factor transformador principal es el hombre, para quien se genera y crea el concepto, primeramente como una esencia, y luego con sentido comunitario de participación. Esto induce a la consideración de que el desarrollo endógeno como tal, representa una tendencia propulsora a un cambio, pero, a su vez, el factor de mayor interés para promoverlo, es el hombre.

Durante el siglo XIX y comienzos del XX, Venezuela se dedicaba principalmente a la explotación del café y el cacao para la exportación, esto generó un estilo de vida europeizante, según Martínez (2006), lo cual significaba el cambio de una mentalidad venezolana para mantener el dominio humano y territorial mientras se consumía todo lo que era europeo como productos e ideas.
Posteriormente, Venezuela, entra en la etapa de la explotación de los hidrocarburos por empresas transnacionales en su mayoría de concesión norteamericana; esto no generó cambios estructurales económicos que impulsasen un crecimiento del país, por cuanto no hubo planes de inversión interna, ni siquiera para incrementar un aparato productivo que generase bienestar en la población, en vivienda o en educación, entre otros.

Hoy Venezuela debería dar respuesta a esta situación e impulsar un nuevo modelo de desarrollo donde el hombre y sus necesidades sean la base fundamental; a este respecto, de acuerdo con Barrios (citado por Castellano, 2005), en años recientes se ha empezado a valorar las iniciativas locales de desarrollo y creación de empleo como una vía novedosa para promover el crecimiento económico y el bienestar social de un ámbito subnacional dado. Por estas razones el gobierno nacional de turno ha impulsado un nuevo proyecto que permite a las comunidades excluidas generar su propio progreso a través de la economía social, donde lo cultural es parte importante para el mantenimiento de su entorno así como su identidad, su pasado histórico y su modo de producción. Por ello la configuración de estas unidades de producción deben estar diseñadas por los mismos agentes involucrados; donde confluyan los distintos saberes que circulan entre sus miembros, los productores, los hombres y mujeres para transformar su hábitat en un núcleo de desarrollo y se cambie de una mirada particular de la realidad local a la generación de una mirada global del mundo.

Dentro de estos actores involucrados deben estar presentes organismos 
para capacitar y brindar apoyo a estas comunidades, las cuales no deben ser vistas como objetos de cambio sino sujetos que están en un proceso de transformación que necesitan ser adiestrados para que en un futuro próximo no dependan de agentes externos (Martínez, 2006).

Sunkel (1991) señala que un verdadero desarrollo nacional y regional tendrá que basarse primordialmente en la transformación de los recursos naturales que la América Latina posee en relativa abundancia, en el aprovechamiento mesurado y eficiente de la infraestructura y capital acumulado, en la incorporación del esfuerzo de toda su población -en especial aquella relativamente marginaday en la adopción de estilos de vida y consumo, técnicas y forma de organización más apropiada a ese medio natural y humano.

Para este autor, llevar a cabo exitosamente ese proceso de transición implica, sin lugar a dudas, superar la etapa de desarrollo hacia adentro y las experiencias más unilaterales de crecimiento hacia fuera para encaminarse hacia una futura estrategia de expansión e industrialización "desde adentro", portadora de un dinámico proceso de acumulación, innovación y aumentos de productividad.

Como se puede apreciar, la visión del modelo se sintetiza de acuerdo a los siguientes criterios: un cambio social que va de adentro hacia fuera, el sujeto transformador con principios y valores que le permita integrarse a las comunidades como ente impulsador, con la idea de avanzar hacia el mejoramiento de la calidad de vida y promoción del desarrollo social y cultural, sin exclusión de la mano obra calificada y comunitaria.
Estos criterios se consolidan, aunado a recursos económicos, políticas estadales, participación ciudadana, entre otros, como los factores claves integradores del modelo de desarrollo endógeno, cuyo éxito se sustenta en el conocimiento que tengan los ciudadanos de su estructura y dinámica, así como en la manera de utilizar y dominar los recursos informacionales en pro de la integración del ciudadano a la dinámica social, a fin de contribuir a una mejor calidad de vida.

En este sentido Martínez (2006) plantea que existen tres premisas fundamentales para generar desde adentro las condiciones para la transformación, como son: el nuevo papel del estado, la necesidad de un proyecto nacional y el desarrollo endógeno; se deben diseñar las propias estructuras económicas buscando equilibrar los aspectos que integran la constitución de una ciudadanía de contenido social, la búsqueda de la equidad y el rescate de lo público, como espacio para el ejercicio de la democracia, sustentado en la participación a través de la organización. Por ello, la nueva estructura social debe canalizar una nueva clase social articulada entre los diferentes agentes para el cambio: Gobierno, Instituciones y Comunidades; los tres constituyen una nueva clase articulada. De modo que, el camino hacia la expansión necesita un consenso entre el sector público y privado, entre organizaciones de trabajadores, empresarios y organismos estadales y municipales.

La política de desarrollo endógeno tiene una gestión descentralizada que se hace operativa a través de las organizaciones intermediarias que prestan servicios reales y financieros a las empresas y 
Recursos informacionales, desarrollo endógeno y participación ciudadana... Fernández, Norcka; Belloso, Nora y Delgado, Francys

organizaciones. No se trata de facilitar fondos a las empresas, sino de dotar a los sistemas productivos de los servicios que las empresas demandan para mejorar su competitividad en los mercados; y a la sociedad con los medios que favorezcan una mejor calidad de vida. En consecuencia se requiere la participación del estado para que a través de sus actividades estratégicas le preste apoyo a este modelo de desarrollo.

En la actualidad, en algunos países del mundo el estado cumple un papel activo en crear, modelar y guiar, incluyendo la promoción de nuevas tecnologías. Este rol como entrenador requiere de una contextualización y planificación en cada una de las dimensiones que participan en el cometido. Así como diseñar estrategias en materia pública propia para la actividad endógena en las diferentes dimensiones como: económica, ética, tecnológica, cultural, organizacional, social y militar (Mas, 2005).

Para Vásquez (citado por Escobar, 2006) cuando las políticas las hacen los actores locales, es decir, la comunidad local organizada para tratar de resolver sus problemas, se puede obtener una mejor calidad de vida, erradicando la pobreza, mejorando el bienestar individual y colectivo y obteniendo inversiones en bienes y servicios, además de una infraestructura óptima para el desarrollo.

Sin embargo, Cuadrado (2006) señala en su teoría del desarrollo endógeno que las políticas regionales deben descentralizarse a través de las siguientes acciones: apoyo a las iniciativas empresariales locales, incentivos (fiscales, monetarios y, sobre todo, administrativospolíticos), incentivos reales (terrenos, servicios a la empresa, infraestructuras locales) a la creación de empleos, formación de profesionales, aumento del gasto en educación y difusión de nuevas tecnologías.

De esta manera, la base de sustentabilidad política sobre la cual se erige el Desarrollo Endógeno es la existencia de una comunidad política, comprometida y preparada para apoyar a la sociedad en su despegue y recorrido hacia la visión propuesta. Esto supone la capacidad política de la totalidad social para autogobernarse.

Aún cuando el gobierno nacional es el principal responsable de promover políticas que equilibren la distribución del patrimonio entre los municipios, la regulación así como la prestación de servicios públicos, la formulación de políticas y programas de fomento económico local y regional además de velar y tomar medidas para mantener la estabilidad macroeconómica. La municipalidad debe contribuir al desarrollo económico del municipio, para mejorar el nivel de vida de la población, su función es ser promotora, facilitadora y reguladora de la integralidad del desarrollo. Es la responsable de las políticas públicas municipales y de generar condiciones favorables para el desarrollo productivo en el municipio. Esto implica mejoramiento de los ingresos de sus habitantes así como en su nivel de vida.

Si bien es cierto que todos los elementos que integran el modelo de desarrollo endógeno son importantes, la participación de la gestión municipal lo es más aún, por cuanto va a servir de enlace en la promoción del proyecto, como el facilitador de los recursos y gran apoyo comunitario. El municipio es el ente pro- 
motor y controlador para garantizar tanto la ejecución de los programas, como el aporte económico y los recursos informacionales.

\section{Participación ciudadana: consideraciones generales.}

La implementación de una política nacional de información, vista como el conjunto de estrategias que propicien el desarrollo y el reposicionamiento de este sector, constituye una tarea compleja que requiere de la participaron de todos los sectores de la vida socioeconómica; para que esta implementación sea valiosa, es preciso partir del contexto político concreto del país de su desarrollo socioeconómico y de aspectos históricos culturales entre otros.

Por lo cual, la tendencia hacia el fortalecimiento de la relación estado sociedad local presupone una mayor articulación entre este último y municipio a partir del efectivo fortalecimiento de dichas organizaciones. Apareciendo así, el desafío para los gobiernos municipales de tratar de articular sus acciones con la sociedad organizada, fortaleciendo sus vínculos, a fin de ampliar el modelo de gestión municipal y de desarrollo endógeno hacia algo más que solo el equipo de gobierno de turno.

Hoy en día el concepto tradicional de ciudadanía que reconoce derechos individuales, tales como derechos políticos, libertad de pensamiento y otros, está en crisis ya que la idea de ciudadanía se ha fundamentado en la afirmación que todos los individuos son libres e iguales por nacimiento, relacionando el estatus legal que establece los derechos del individuo frente al estado.
Sin embargo, este concepto tradicional se enfrenta a reivindicaciones en pro de derechos colectivos a partir de nuevas identidades como son el género, la edad, la migración o las etnias, entre otros. Según Sanhuesa (2004), las demandas de derechos de diferentes grupos que presionan al estado para la incorporación de sus identidades o demandas particulares en las políticas públicas, ratifican que el ciudadano ya no es el receptor pasivo de derechos que goza de la protección de la ley, sino que se identifica con la intervención del estado.

De tal manera, pensamos que la ciudadanía constituye, entonces, un principio articulador que afecta las diferentes posiciones del sujeto en su rol de agente social al tiempo que permite el respeto a la libertad individual, cuestión esta refrendada por Arroyo (1999), al señalar que la participación ciudadana apunta hacia una transformación que imponen las demandas de la sociedad, tanto en lo que concierne al desarrollo de una representación social capaz de controlar y presionar a la gestión pública, como en la búsqueda de espacios sociales, en los cuales los individuos logren producir actividades que expresen sus necesidades.

La participación ciudadana representa la relación estado y sociedad civil; o más concretamente, es la intervención de los particulares en actividades públicas en tanto portadores de intereses sociales, Sanhuesa (2004:2). También puede ser vista como el proceso de intervención de la sociedad civil, individuos y grupos organizados en las decisiones y acciones que los afectan a ella y a su entorno.

La participación ciudadana apunta hacia una transformación que imponen 
Recursos informacionales, desarrollo endógeno y participación ciudadana... Fernández, Norcka; Belloso, Nora y Delgado, Francys

las demandas de la sociedad, tanto en lo concerniente al desarrollo de una institucionalidad de representación social capaz de presionar y controlar al gobierno, como en la búsqueda de espacios sociales en los cuales los individuos logren producir actividades que expresen su necesidad (Spinelli, 2000).

De acuerdo a lo planteado por los diferentes autores, a nuestro juicio la participación ciudadana se ubica en la existencia de un poder público que pretende ser la expresión del interés general de la sociedad y de una administración que realiza la orientación del mismo; ésta se debe fomentar en todos los niveles del poder público. Sin embargo, debe estar claro que cada nivel de gobierno exige una estrategia diferente de participación. El espacio del nivel local, municipal, ha sido el punto de partida pero hay que buscar incidir en el nivel nacional, deslindando los niveles y estrategias de participación mas apropiados para cada uno.

Según Nikken (1994:18) "los derechos humanos son inherentes a las personas y su existencia no depende del reconocimiento de un Estado, siempre es posible extender el ámbito de la protección a derechos que anteriormente no gozaban de la misma".

Partiendo de esta afirmación, la Red de Apoyo por la Justicia y la Paz de la Universidad Católica Andrés Bello (2002) estableció los siguientes fines de la participación ciudadana:

- Fortalecer la democracia basada en el principio de la soberanía popular y de conformidad con los derechos humanos.
- El desarrollo individual y colectivo de las personas en ámbitos como el social, político y familiar entre otros.

- Consolidar una sociedad democrática, pluralista, tolerante, participativa, crítica, libre, solidaria y protagónica.

- Fomentar las diversas formas de organización social.

- Fomentar el efectivo control ciudadano en los asuntos públicos a través de un adecuado suministro de información por parte del estado.

- Establecer la educación como un componente indispensable para el ejercicio de la participación.

- Consolida a la solidaridad social como eje de la participación en todos los ámbitos del poder público.

También señalan los autores, que la participación ciudadana se basa en principios entre los cuales podemos mencionar: es un derecho humano, transparente, interdependencia, voluntaria, solidaridad social, educativa, complementariedad, pluralidad, no discriminación, corresponsabilidad, deliberación pública, control de la gestión pública, reconocimiento a todos los autores sociales, diversidad de medios y mecanismos, autonomía, la información debe ser plena, permanente y totalmente accesible, valoración de los saberes populares, su limite son los derechos humanos, inclusiva no exclusiva.

Todos estos principios y fines tienen como punto central la sustitución de una democracia representativa por una democracia participativa, lo que incide en el impacto del desarrollo endógeno y en convertirse en un componente esencial de un modelo alternativo de gestión so- 
cial más justa y democrática, por lo cual se asocia estrechamente con la modernización del estado. Si por gestión pública se entiende la manera de organizar los recursos entre ellos los informacionales para el cumplimiento de los objetivos y tareas del estado, la participación ciudadana en la gestión pública se refiere al rol del ciudadano en cuanto la participación y utilización de las decisiones y gestiones asociadas a la implementación de acciones públicas. Obviamente la gestión pública local, posee atribuciones muy particulares, entre estas se encuentran actuar e interactuar en una realidad compleja y figurar como gestor democrático de la participación ciudadana en la búsqueda de metas comunes de bienestar económico y social; pero quizás la mas importante sea la de favorecer y promover el desarrollo local.

Al decir de Cunill Grau (1997), la participación efectiva de la mayoría de la población en forma de ciudadanía democrática, requiere de un acceso de todos a los recursos de la sociedad y modalidades de organización y participación política que estén enraizadas en las condiciones propias de esa sociedad.

Por lo tanto, la participación ciudadana implica a nuestro juicio la representación en condiciones de igualdad en pluralismo político y oportunidad de deliberación, lo que supone la identificación con las claves normativas de la participación política: igualdad política, pluralismo político, deliberación pública y un compromiso con la libertad. Todo ello converge a una nueva concepción y articulación entre estado y sociedad, llevando al estado a propuestas de democracia directa y participativa y en una mayor eficiencia de prestación de servicios públicos y, por ende, del desarrollo endógeno, entendiendo que éste es la habilidad para innovar al nivel local, ya que el desarrollo regional implica un proceso de cambio estructural localizado, asociado a un permanente progreso de la región, de la sociedad que la habita y de cada individuo de ella.

Es por ello que el Estado venezolano debe garantizar el derecho a la participación según los señalan los instrumentos jurídicos internaciones y la Constitución de la República Bolivariana de Venezuela (CRBV 1999), y en ningún caso puede pretender a través de sus órganos, una reversión en estos contenidos del derecho que han sido reconocidos como inherentes a la persona humana. La participación real de los ciudadanos no se dará si el estado no promueve los mecanismos que la hagan posible; la participación requiere consolidar espacios legítimos y formas de intercambio entre ciudadanos y ciudadanas y los órganos del poder público. De tal forma que, el estado debe crear o mantener los espacios concretos de participación y cuidar que se garanticen en todas las estructuras del poder público; entre otras en las siguientes: en el poder público nacional, contralorías, concejo federal de gobierno; en el poder público estatal: concejo de planificación y coordinación de políticas; en el poder público municipal: gestión, creación de parroquias, concejo local de planificación, y otros nuevos sujetos de la descentralización Art. 70 de la CRBV, Universidad Católica Andrés Bello (2002).

Entre los organismos internacionales que rigen esta materia se encuentran: 
Recursos informacionales, desarrollo endógeno y participación ciudadana...

Fernández, Norcka; Belloso, Nora y Delgado, Francys

- Comité de Derechos Humanos de las Naciones Unidas (CDH). Observación general 25 del comité del 12-0796 que señala el derecho a participar en asuntos públicos, derecho a votar y derecho a las funciones públicos en condiciones de igualdad.

- Pacto Internacional de Derechos Civiles y Políticos (PIDCP) el cual establece "... que el pacto impone a los Estados la obligación de adoptar las medidas legislativas o de otro tipo para garantizar que los ciudadanos tengan efectivamente la posibilidad de gozar de los derechos que ampara", Art. 5.

- La Declaración Universal de Derechos Humanos (DUDH) que señala que en el artículo 21 y 30 la participación política de los ciudadanos.

- La Declaración Americanas de Derechos Humanos (DADH) Art. 20.

- Pacto Internacional de Derechos Civiles y Políticos (PICP) Art. 25.

- Convención Americana de Derechos Humanos (CACH) Art. 23 y 29.

- Pacto Internacional de Derechos Económicos, Sociales y Culturales (PIDSC) Art. 15.

En el ámbito nacional la Constitución de la República Bolivariana de Venezuela establece protección constitucional en asuntos públicos, ya no solo en su dirección sino, expresamente en otros sectores tales como: formación, ejecución y control de la gestión pública (Art. 62), participación en campos específicos como salud (Art. 48), seguridad social (Art. 86), educación (Art. 102), ordenación del territorio en materia electoral (Art. 294), discusión y aprobación de leyes (Art. 211).
Además especifica las maneras de participación política e incluye la participación en los ámbitos políticos, económicos y sociales (Art. 70).

Por otra parte el encabezado de la CRBV señala "En nombre y representación del pueblo soberano..." para transformar el Estado y crear un nuevo ordenamiento jurídico que permita el funcionamiento efectivo de una democracia social y participativa y el Art. 6 indica "El gobierno de la República Bolivariana de Venezuela y de las entidades políticas que la componen es y será siempre democrático y participativo...".

Cabe destacar la relación que existe entre el sector información, ciudadanía y desarrollo endógeno, la información como un principio de la participación ciudadana debe ser permanentemente y totalmente asequible, debe existir disponibilidad de la información, es decir, el acceso a la información en el momento en que cualquier individuo u organización la estime necesaria. Ya que los ciudadanos también participan en la dirección de los asuntos públicos ejerciendo influencia mediante el debate y el dialogo público con sus representantes y gracias a su capacidad para organizarse. Esta participación se respalda garantizando la libertad de expresión, reunión y observación.

El Informe Anual de la Convención Interamericana de Derechos $\mathrm{Hu}$ manos (2000:21) señala "...el titular de la información es el individuo que delegó en los representantes el manejo de los asuntos públicos: El principio de transparencia lo que demanda es una posición servicial de la Administración, aportando aquella documentación que hubiera sido previa, correcta y clara- 
mente solicitada en la medida que no se encuentre temporalmente excluido el ejercicio del derecho". Sin esta información no puede ejercitarse plenamente el derecho a la libertad de expresión como un mecanismo efectivo de la participación ciudadana, ni de control democrático de la gestión gubernamental.

\section{Vinculación entre recursos informacionales, desarrollo endógeno y participación ciudadana.}

Considerando el desarrollo que ha tenido la información tanto en las últimas décadas del siglo $X X$ como en los inicios del siglo XXI, es importante destacar que ella como tal, constituye una herramienta importante tanto a nivel del proceso de aprendizaje como, a su vez, el medio idóneo para adquirir conocimiento, y generar nuevamente información necesaria para los procesos de cambio, sobretodo en la necesidad que tiene la comunidad de saber, para tener mayor participación comunitaria.

No puede concebirse el desarrollo endógeno, por ejemplo, si la comunidad no dispone de herramientas de aprendizaje, que permitan participar con la suficiente información, fuerza y recursos, para crecer, participar con seguridad y entero dominio de la situación a la cual se enfrenta. La información a criterio de Lau (2002:78), "constituye un medio transportador del conocimiento desempeña un papel vital en el autoaprendizaje por lo tanto las habilidades informativas son necesarias..." Esto significa, que para llevar a cabo el desarrollo endógeno, es fundamental estar informado y hacer buen uso de los recursos informacionales disponibles. Es una necesidad fundamental para los propósitos que requiere tanto el desarrollo endógeno así como la participación ciudadana. Orna y Graham (2001: 24), consideran que información es la forma visible o audible en que transformamos nuestro conocimiento cuando deseamos comunicarlo a alguien".

Cabe destacar, que la información constituye un medio que enlaza dos elementos importantes: el desarrollo endógeno y la participación ciudadana. Ambas necesitan el hilo conductor, representado por la información, la cual puede manifestarse por diversas vías: tecnológicas, físicas. Puede convertirse a su vez en productos electrónicos, digitalizables (texto, imagen, voz), que prestarían un apoyo importante a la comunidad.

Ahora bien, el punto central está plasmado en todo lo que represente seguridad para que tanto el desarrollo endógeno, como la participación ciudadana no se vean afectados. Esto se concibe primeramente al derecho que tienen todos los ciudadanos de estar informados inclusive con las mismas oportunidades. Por otra parte el recurso informacional como producto, es una necesidad para el usuario, el querer generar mayor desarrollo, no se concibe en forma aislada, se requiere de sistematización, de alertas como vía rápida de sustento, de interactuar y manifestar un sentir ciudadano.

La complejidad de los cambios sociales, tecnológicos a los cuales está sometida la sociedad en el despertar del siglo XXI, compromete tanto a los gobernantes como a la comunidad, a la búsqueda de alternativas que le permitan una solución viable a los problemas y a las opcio- 
Recursos informacionales, desarrollo endógeno y participación ciudadana... Fernández, Norcka; Belloso, Nora y Delgado, Francys

nes de actividades productivas, para un mayor crecimiento comunitario. De estas consideraciones entre otras, surge la participación comunitaria y por consiguiente una mirada hacia el interior de las comunidades, como lo es el desarrollo endógeno, que le permitan en forma sustentable obtener beneficios en el corto plazo. Para ello, es necesario que las comunidades se organicen e instruyan, a objeto de enfrentar con éxito las bondades que presenta tanto el desarrollo endógeno, como la participación comunitaria.

Con relación a esta necesidad que tiene el usuario son muchas las formas de obtener conocimiento. Producto de ello, son los recursos informacionales. Éstos pueden alcanzarse a través de medios físicos como las bibliotecas, los archivos, los centros de información y básicamente los productos que de ellos se derivan.

Asimismo, la presencia de la tecnología en su amplio radio de acción, viene a facilitar el procesamiento de los datos, lo cual hace más atractivo la utilización de la información para las comunidades. "El aprovechamiento de las tecnologías de información radica en las constantes que en lo futuro se mantengan dentro del sector de servicios de información..." (Lafuente, 1999:90). Es importante que tanto los usuarios, como las comunidades utilicen al máximo los recursos, para el logro de bienes. Sin embargo, surge la siguiente interrogante: ¿realmente los ciudadanos utilizan los recursos informacionales para lograr la participación ciudadana en el desarrollo endógeno local?, ¿brinda el municipio los recursos informacionales necesarios para lograr esto?

De igual manera, Morales (1999) plantea que para que el derecho a la infor- mación se cumpla, es suficiente que exista una armónica relación entre sociedad y el Estado. Estos ámbitos tienen que interactuar uno y otro para que desde la localidad se tomen en cuenta los nuevos comportamientos, actitudes y valores de la sociedad globalizada y se advierta el vaIor estratégico del conocimiento, adquirido a través de los recursos informacionales como elemento esencial para el desarrollo de las sociedades. Ésto implicaría alianzas estratégicas entre la gestión pública y la comunidad, a través de su participación, para la generación de procesos de desarrollo social.

No es posible visionar el éxito en el desarrollo de proyectos comunitarios, sino se tiene interés por el uso y conocimiento de productos informacionales, que contribuyan al mejoramiento de proyectos institucionales. Tanto las alcaldías, como las comunidades, necesitan conocimiento de nuevas alternativas, de opciones viables y productivas, pero si no se disponen de productos que le den celeridad al proceso, es probable que no se logren los objetivos previstos, y se incurra en pérdidas lamentables, tanto de los recursos humanos, como en el tiempo de ejecución y disposición para el desarrollo productivo desde adentro.

Para Primera et al. (1999), la idea de reorientar las políticas para que los recursos informacionales de los que dispongan o puedan disponer la gestión pública puedan utilizarse para fines de desarrollo local, comenzaría a cobrar fuerza al evidenciarse la existencia de iniciativas, decisiones, ideas y estrategias; plasmadas en planes, programas, proyectos y otros medios que plantean avances en diversas áreas. 
El triangulo informacional circunscrito a la participación ciudadana, desarrollo endógeno y fuentes gubernamentales locales, viene a ser el punto fundamental que las nutre. Conforma el núcleo a través del cual se sustentan los elementos que integran el triangulo, la desarticulación de cualquiera de estos elementos con el centro, en este caso, los recursos informacionales, puede producir ruptura e impedir el desarrollo del proyecto. La luz del éxito está en la utilización eficiente de los recursos informacionales. Para ello, es necesario, generar, instruir, difundir, evaluar y posteriormente controlar, para capitalizar el esfuerzo en su utilización.

Sanhuesa (2004) expone que la participación aumenta la comprensión del quehacer público. Facilita la transparencia y el control social. La participación de la ciudadanía contribuye a la redefinición de lo público, quitándole el uso exclusivo de lo público a lo estatal. Es decir, que la gestión pública involucra a la sociedad civil, crea espacios de participación y contribuye a corregir fallas, así como a construir y reconstruir organizaciones.

Dentro del manejo de los recursos informacionales como guía orientadora y facilitadora para la toma de decisiones, se están buscando opciones más directas y de mayor utilidad que permitan a las comunidades y a la municipalidad ofrecer alternativas, para la participación ciudadana en pro de crear conciencia y herramientas al colectivo. En este sentido, es importante acotar que se está trabajando con una herramienta denominada prototipo de información municipal. Ésta, permitirá organizar la información estadística municipal de forma tal, que los actores locales (autoridades y comunidades organizadas), puedan elaborar índices cuantitativos (Ministerio de Planificación y Desarrollo, 2006). Esta información va a constituir un importante insumo para la planificación, organización y control de todo cuanto se proponga a nivel comunitario para el desarrollo endógeno y la participación ciudadana. Para algunos autores (Sanhuesa, 2004 y Garretón, 2004) la participación ciudadana es un pilar del fortalecimiento de la democracia y de la gobernabilidad democrática, permite estar más cerca del ejercicio de la responsabilidad pública e incluso tener responsabilidad en ella. Aumenta la comprensión del quehacer público y el conocimiento de lo que hace realmente. Facilita el control social y se involucra en todas las etapas de la gestión pública local.

Es por ello que existe una vinculación entre el desarrollo endógeno (cuando el municipio establece políticas, planes y proyectos), participación ciudadana (rol del ciudadano en la gestión pública) y recursos informacionales (información que nutre a los dos elementos anteriores) tal como se muestra en el cuadro de la siguiente página.

\section{Conclusiones}

Del presente trabajo se infieren las siguientes conclusiones:

Los recursos informacionales se vinculan con el desarrollo social, siempre y cuando su acceso y aprovechamiento se oriente a las realidades de una localidad determinada y se impulsen hacia el progreso, a fin de responder a nuevos 
Recursos informacionales, desarrollo endógeno y participación ciudadana... Fernández, Norcka; Belloso, Nora y Delgado, Francys

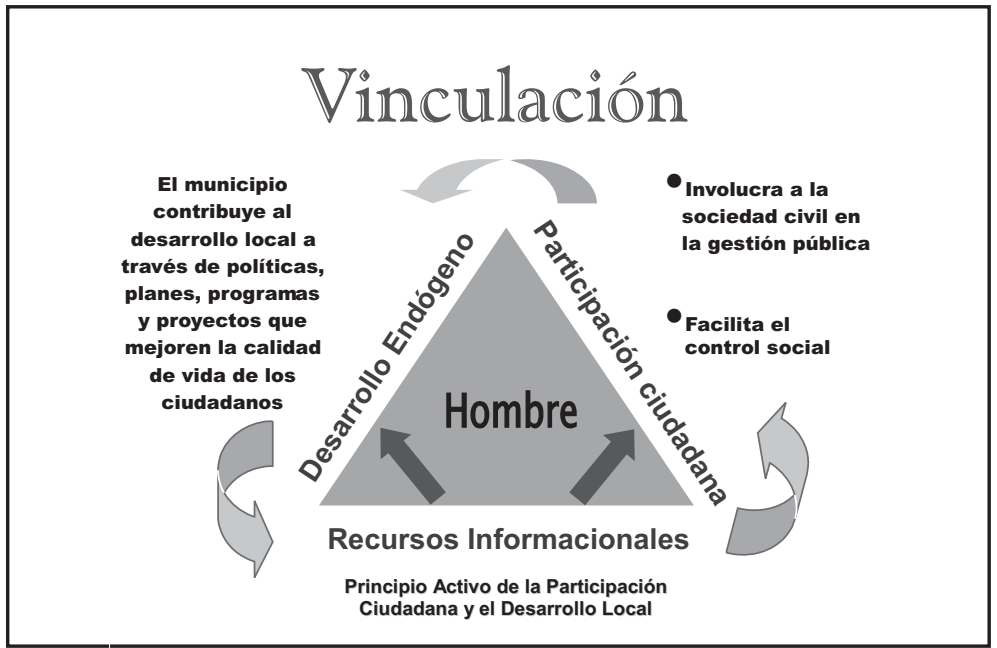

Fuente: Elaboración propia.

modos de producción y de relación entre el estado y la sociedad. Es importante que las políticas diseñadas en esta temática sean dirigidas desde el ámbito local, por ser el nivel de gobierno más cercano a la realidad comunitaria. Así como, la generación de mecanismos para la participación ciudadana.

En este sentido, se puede concluir que el estado venezolano ha promulgado leyes y ha formulado políticas, planes, programas y proyectos dirigidos a impulsar los recursos informacionales en el país, con el propósito de disminuir la exclusión social de las comunidades.

A pesar de esto, los lineamientos en materia informacional no se ajustan a la realidad de los municipios venezolanos. Lo cual, dificulta el alcance de los planes, programas y proyectos que se emprendan.
La gestión pública puede involucrar a la sociedad civil en procesos de participación y contribuir en la resolución de problemas a través del uso de los distintos recursos informacionales, puesto que permiten dar a conocer a los ciudadanos la información que la gestión pública lleva a cabo, permitiendo participar en los procesos de desarrollo local.

A partir de la investigación realizada se ha evidenciado que es conveniente involucrar a los ciudadanos en la gestión pública cuando estos expresan interés en participar. Sin embargo, deben ser orientados e informados sobre cómo deben efectuar su participación.

La participación ciudadana puede constituir una contribución sustancial a las acciones efectuadas por la gestión pública local a través de la utilización de los recursos informacionales diseñando proyectos dirigidos hacia el desarrollo endógeno en esa comunidad. 


\section{Referencias bibliográficas}

Arroyo, Daniel (1999). La Descentralización y la Participación Ciudadana en la Gestión Local. Recuperado el 27.11.2005 desde: http://www.ubiobio.cl/cps/ponencia/doc/p18.4.htm

Asamblea Nacional. República Bolivariana de Venezuela. La Constitución (1999). Segunda Versión: Gaceta Oficial No. 5453del 24-Mar-2000.

Castellano B., Hercilio (2005). La planificación del desarrollo sostenible. CENDES. Caracas. Venezuela.

Cornella, Alfons (2003). Gerencia de los Recursos de Información. Recuperado el 04.11.2005 desde: http://eprints.reclis.org

Coutin, Adrián (2005). Programa para el Establecimiento de la Gerencia de Recursos de Información en las Organizaciones. Recuperado el 06.04.2006 desde: http://eprints.reclis.org

Cuadrado Roura (2006). Desarrollo Endógeno. Ministerio de Hábitat y Vivienda, disponible en http://www.mhv.gob.ve/ habitat/pag/endogen.php (Consulta: 2006, julio 28).

Cunill Grau, Nuria (1997). Repensando lo público a través de las sociedad. Venezuela Nueva Sociedad.

Currás, Emilia (2001). Caos y Orden en la Organización del Conocimiento. Ciencias de Información. 27 (4) 239-259.

Escobar, Fernando (2006). Morrocoy debe ser declarado patrimonio turístico de la comunidad. Disponible en http://www.tiempo.uc.edu.ve/tu514/ paginas/4.htm. (Consulta: 2006, agosto 04)

Esteban, Miguel Ángel (2005). El Programa de Gestión de los Recursos de Información en la Organizaciones. Departamento de Ciencias de la Documentación. Universidad de Zarago- za. Recuperado el 15.12.2005 desde: http://tramullas.com

Garretón, Manuel (2004). Redefinición de Gobernabilidad y Cambio Político. Universidad de Chile. Asociación Chilena de Ciencias Políticas.

Goñi, Ivis (2000). Algunas Reflexiones sobre el Concepto de Información y sus Aplicaciones para el Desarrollo de las Ciencias de la Información. AClMED, 8 (3) 201-207. [Versión Electrónica]. Recuperado el 20.11.2005 desde: http://bvs.sld.cu/revistas/aci/vol8_ 3_00/aci02300.htm

Guerrero, Roberto (2004). Recursos de Información Corporativos en las Áreas de Servicios de la Biblioteca Nacional de Ciencia y Tecnología. Recuperado el 20.10.2005 desde: http:// eprints.rclis.org

Instituto Latinoamericano y del Caribe de Planificación Económica y Social ILPES (2006). Disponible en: http:/ /www.infomipyme.com/Docs./ GT/sidel/municipal/ConsideracionesConceptuales.htm. (Consulta: 2006, agosto 04).

Informe Anual de la Convención Interamericana de Derechos Humanos, (2000) Vol. II. Disponible en: www.ucab.edu. Ve/ucabnuevo//edh/recursos/ib_parti.doc. (Consulta: 2006, julio 26).

Lafuente López, Ramiro (1999). Biblioteca Digital y Orden Documental. México: UNAM, Centro universitario de Investigaciones Bibliotecológicas.

Lau, Jesús (2002). Información: insumo básico del aprendizaje. En Memoria del XIX Coloquio Internacional de Investigación Bibliotecológica y de la Información (pp.51-78) México: UNAM.

Martín, Yolanda (2001). La Documentación de la Unión Europea: Concepto y Clasificación. Biblioteconomia i Documentació. (7) desembre, 2001. [Versión Electrónica]. Recuperado el 
Recursos informacionales, desarrollo endógeno y participación ciudadana...

Fernández, Norcka; Belloso, Nora y Delgado, Francys

07.11.2005 desde: http://www.ub. es/bibllio/bid/07marti2.htm

Martínez, Juan Antonio (2000). Naturaleza y Cualidades del Documento en Archivos, Bibliotecas, Centros de Documentación y Museos. Revista de Investigación lberoamericana en Ciencia de la Información y Documentación. 1 (1): 11-16.

Martínez, Juan Antonio (2001). Teoría de la Información Documental y de las Instituciones Documentales. $2^{2} \mathrm{Ed}$. Madrid: Mio-Cid.

Martínez, Edgar (2006). Tips antropológicos sobre el desarrollo endógeno para configurar una nueva clase. Disponible en: http://www.rebelion.org/noticiaphp?id=33341. (Consulta 2006, agosto 4).

Mas, Maria (2005). Desarrollo Endógeno. Cooperación y Competencia. Editorial PANAPO de Venezuela C. A. Caracas. Venezuela.

Ministerio de Planificación y Desarrollo (2006). Economía Social y Desarrollo Endógeno. Disponible en: http://www. gerenciasocial.org.ve/diat/areas_trabajo.htm. (Consulta 2006, julio 27).

Morales, Estela (1999). El derecho a la Información y las Pólíticas de Información en América Latina. Ponencia presentada en la 65th Conferencia de la IFLA Bankok, Tailandia. Recuperado el 07-01-2006 desde http://wwwifla.org/IV/ifla65/papers/0561375htm.

Nikken, Pedro (1994). El Concepto de Derechos Humanos Antología. Básica en Derechos Humanos. IIDII, Costa Rica.

Orna Elizabeth y Graham, Stevens (2001). Cómo usar la información en trabajos de investigación. España: Editorial Gedisa.

Peña, Dionnys (2004). Políticas de Información en la Orientación de los Sistemas Informáticos para el Desa- rrollo Local. Trabajo de ascenso para optar a la categoría de profesor agregado. Universidad del Zulia. Facultad de Humanidades y Educación. Escuela de Bibliotecología y Archivología. Maracaibo, Venezuela.

Ponjuán, Gloria (1998). Gestión de Información en las Organizaciones: Principios, Conceptos y Aplicaciones. Santiago de Chile: Centro de Capacitación en Información.

Primera, Nelly; López, Mirtha y Montes de Oca, Yorberth (1999). Modernización de los Sistemas de Información en la Alcaldía de Maracaibo. Recuperado el 8.11.2005 desde: http://72.14. 207.104/

Primera, Nelly; Montes de Oca, Yorberth y Fernández, Norcka (2002). "Informatización al Ciudadano desde el Gobierno Local: Alcaldía de Maracaibo-Venezuela". Revista Venezolana de Ciencias Sociales. 6 (1) 9-25.

Ranguelov, Stanislav (2004). Sistemas para gestionar la Información Organizativa y el Conocimiento. Recuperado el 13.12.2005 desde: http://www.gestiondelconocimiento.com

Sánchez, Bárbara y Graña, Elena (2000). "Apuntes y Reflexiones en Torno a una Política Nacional de Información en Cuba". Anales de Documentación. 8 (3) 186-193. [Versión Electrónica]. Recuperado el 21.10.2005 desde: http://eprints.reclis.org

Sánchez, Bárbara y Rodríguez, José Vicente (2000). La Información como Recurso en el Desarrollo de las Organizaciones de las Administraciones Públicas. Anales de Documentación. (3) 155-165. [Versión Electrónica]. Recuperado el 21.10.2005 desde: http://eprints.reclis.org

Sanhuesa, Andrea (2004). Participación ciudadana en la gestión pública. Santiago de Chile. Corporación participa. 
Spinelli, Graciela (2000). La descentralización y participación ciudadana en la gestión local. Argentina. Universidad de Comanhuerequen, Disponible en http://www..ubiobo.cl/cps/ponencia/doc/p18.4htm. (Consulta: 2006, julio 20).

Sunkel, Oswaldo (1991). El desarrollo desde adentro. Un enfoque neoestructuralista para la América Latina. Fondo de cultura económica. México

Universidad Católica Andrés Bello. Centro de Derechos Humanos (2002) Principios que deben orientar la Participación Ciudadana. Caracas. Red de Apoyo por la Justicia y la Paz. Disponible en: www.ucab.edu.ve/ucabnuevo//edh/recursos/ib-parti.doc. (Consulta 2006, julio 26)
Universidad Católica Andrés Bello (2002). Organismos Internacionales que rigen la Participación Ciudadana en: Principios que deben Orientar la Participación Ciudadana. Disponible en: www.ucab.edu.ve/ucabnuevo//edh/recursos/ib-parti.doc. (Consulta 2006, julio 26).

Valdés, Manuela (2000). Consideraciones Generales en torno al Valor Añadido de la Información. Recuperado el 19.10.2005 desde: http://www.bvs. Sld.cu/revistas/aci/vol7_1_99/aci02 199.htm

Vásquez, Barquero (2006). Desarrollo Endógeno en Ministerio de Habitat y Vivienda. Disponible en http://www. Mhv.gob.ve/habitat/pag/endogen. php (Consulta: 2006, julio 28) 